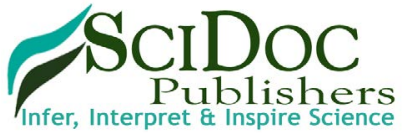

\section{Novel Suturing Technique for Fascia Closure after Laparotomy: Spider Suture to Increase the Tearing Force of the Fascia}

$\operatorname{Demir} \mathrm{R}^{1,2^{*}}$

${ }^{1}$ Department of Surgery, University Hospital Erlangen, Erlangen, Germany.

${ }^{2}$ Medical Care Center, MVZ-Demir, Nürnberg, Germany.

\title{
Abstract
}

Purpose: The objective of this study was to demonstrate a new suturing technique in reference to fascia closure after laparotomy in an experimental setup to increase the tearing force of the fascia.

Methods: Two ordinary DIN A4 papers, which represents the fascia were sutured at the edges in a length of approx. $5 \mathrm{~cm}$ with the known continuous technique in the abdominal surgery and with the new technique, we called it: Spider suture. A bucket arranged on the interconnection was filled with sand until the sutures teared. Measuring the final weight of the bucket allowed comparing the carrying capacities of the paper of the two techniques.

Results: A significant (Fisher's test, $\mathrm{p}=0.017$ ) increase of mean carrying capacity from $1776.5 \mathrm{~g}$ (classic continuous suture) to $5101.75 \mathrm{~g}$ (basic Spider suture) was found. The carrying capacity of the paper was increased to $287 \%$ compared to the classic continuous suture.

Conclusion: Spider suture could be an effective improvement in closure of the abdominal wall in abdominal surgery to minimize the risk of developing of incisional hernia. This method allows the surgeon to perform an abdominal closure adapted to the quality of patient's fascia. Further clinical studies will show the effectiveness of this method.

Keywords: Suture; Abdominal Fascia; Hernia; Laparotomy.

\section{Introduction}

An unsolved surgical problem is the development of incisional hernia after laparotomy. An incidence of 2 to $20 \%$ can be found in literature $[1,2]$. The number of annual laparotomies in the US is 4 to 5 million. Therefore one can expect approximately 500,000 cases of incisional hernia p.a. of which 200,000 need to be treated surgically [1]. In Germany one can expect 40,000-70,000 cases of incisional hernia p.a. Approximate costs for surgery per patient is $5,000-10,000 €$ which accounts for total annual costs of up to 700 million $€[3]$. For suturing the abdominal wall, two techniques are commonly used: continuous suture and simple interrupted suture. The surgeon can choose from resorbing and non-resorbing suture materials. For the resorbing materials there are different time scales for the resorption (the common differentiating factor is half-life).

Despite progress in the development of novel suture materials and the effort of advancing the mentioned suturing techniques, there has been no major improvement of the incidence of incisional hernia after primary fascia closure over the last decades $[2,4]$. Numerous clinical trials disproved the desired effects of novel suture materials.

By thorough analysis of cases of incisional hernia, the author came to the conclusion that though the suture material retains the fasciae, the adjacent tissue slowly tears at the point of maximum stress at the abdominal wall causing the herniae.

In order to avoid incisional herniae, the author saw the need for a novel suturing technique that:

- prevents the fascia from tearing at the puncture sites, - disperses the stress across the whole length of the suture line, and

- Introduces a stress component parallel to the suture line in order to disperse the stress across the two axes parallel and perpendicular to the suture line.

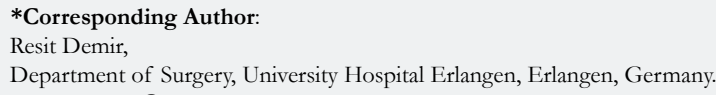

Copyright: Demir $\mathbf{R}^{\circ}$ 2018. This is an open-access article distributed under the terms of the Creative Commons Attribution License, which permits unrestricted use, distribution and reproduction in any medium, provided the original author and source are credited. 
The novel technique could be applied in cases of primary fascial closure after laparotomy, umbilical hernia, incisional hernia when a fascial closure without major increase of intra abdominal pressure is possible or diastasis recti.

\section{Materials and Methods}

Being inspired by a spider web, the novel technique is called Spider suture. Necessary steps for a Spider suture are as follows:

1. A continuous circular suture is applied to the sides of the fasciae and knotted (Figure 3a).

2. A regular continuous suture is applied with the respective puncture sites being outside of the circular suture of step 1 (Figure 3b).

3. Depending on the consistency of the fascia the basic spider suture can be extended by adding further circular sutures in expanding circles (Figure 3c, black dashed lines). Additionally, linear crosslinks can be applied (Figure 3c, red dashed lines).

An experimental setup is presented that allows comparison of the carrying capacities of the novel (basic) spider suture and a state of the art continuous suture:

Two ordinary pieces (Figure 1) of paper were sutured at the edges in a length of approx. $5 \mathrm{~cm}$ with the known technique (Figure 2) and with the technique according to the invention (Figure 3 ). In both cases, monofilament thread ("Resolon 2-0, Resorba") was used. A bucket arranged on the interconnection was filled with sand until the sutures teared (Figure 4). Measuring the final weight of the bucket allowed comparing the carrying capacities of the two techniques. The number of repetitions was 5 for each suture.

Subsequently, the data were incorporated into the IBM SPSS statistics program version 19. The level of significance was determined by the Fisher's test and set at $\mathrm{p} \leq 0.05$.

\section{Results and Discussion}

As expected after continuous suturing technique tearing of the paper started at the stabbed area of the paper. Tearing mechanism in the basic Spider suturing was different. It started also at the stabbed area of the continuous suture, but it stopped at the circular suture. The hole tearing power was dispersed to the circular suture. The tearing was observed as a hole of the sutured part of the paper.

A significant (Fisher's test, $\mathrm{p}=0.017$ ) increase of mean carrying capacity from $1776.5 \mathrm{~g}$ (classic continuous suture) to $5101.75 \mathrm{~g}$ (basic Spider suture) was found (see Figure 5 and Table 1). The main question after primary laparotomy is how to closure the

Figure 1. Experimental setup: Two sheets of ordinary paper as an example of a severed fascia presented by the hemline of both sheets. The suturing area is marked by dotted lines $5 \mathrm{~cm}$.

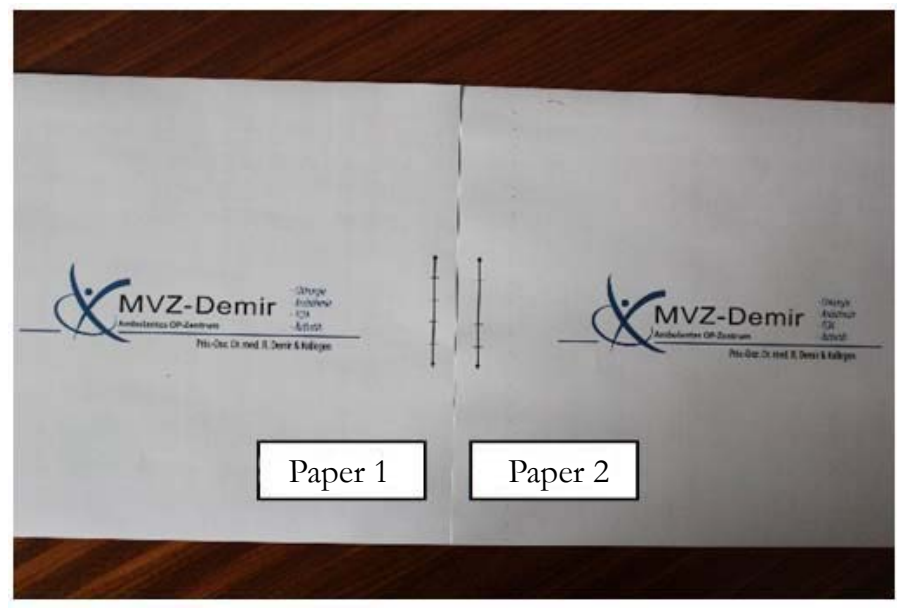

Figure 2. Example for a state of the art suturing technique: continuous suture.

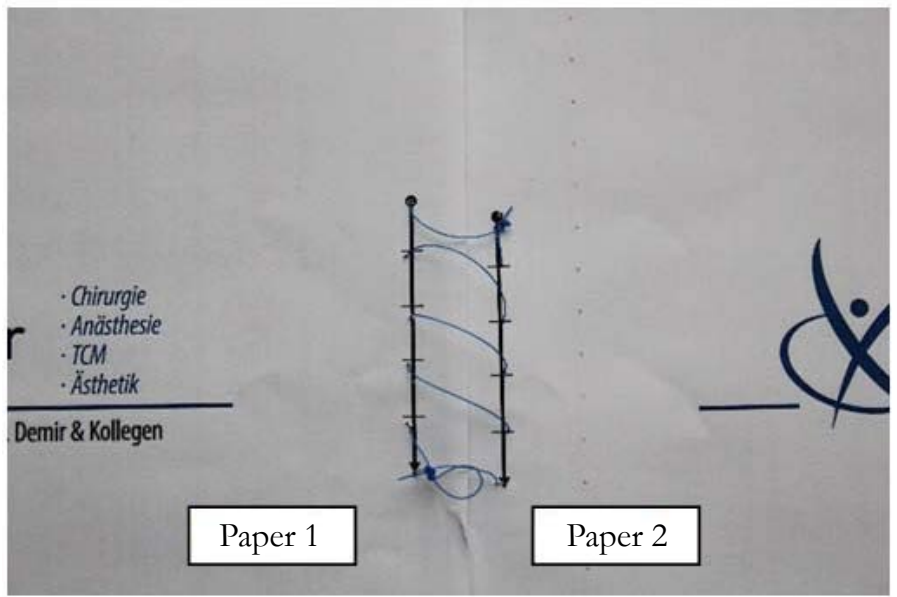


Figure 3. Basic Demir Spider suture: a) circular suture, b) Continuous suture with puncture sites outside of the circular suture. C) Extended spider suture: additional circular sutures depending on the consistency of the fascia (dashed lines 1 and 2) and crosslinks if necessary (red lines).
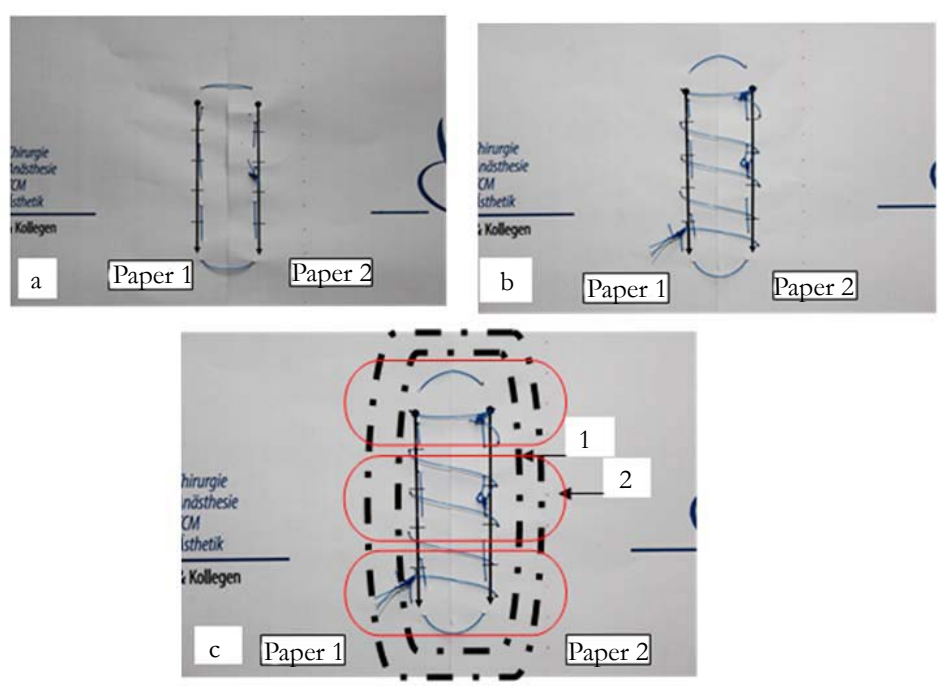

Figure 4. Ordinary Paper was fixed between two loungers. A bucket arranged on the interconnection was filled with sand until the sutures teared. Measuring the final weight of the bucket allowed comparing the carrying capacities of the two techniques.

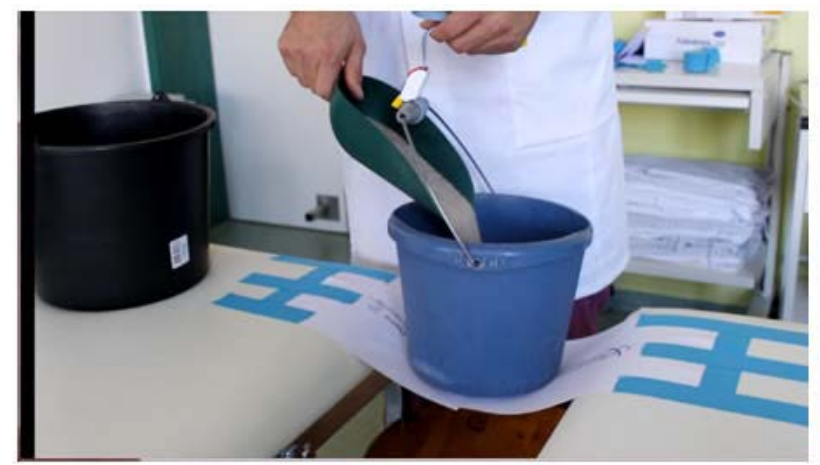

Figure 5. Comparison of carrying capacity of the two suturing techniques (Fisher's test, $\mathrm{p}=\mathbf{0 . 0 1 7}$ ). The tear force of the paper was increased to $287 \%$ significantly, when performed the Spider suture.

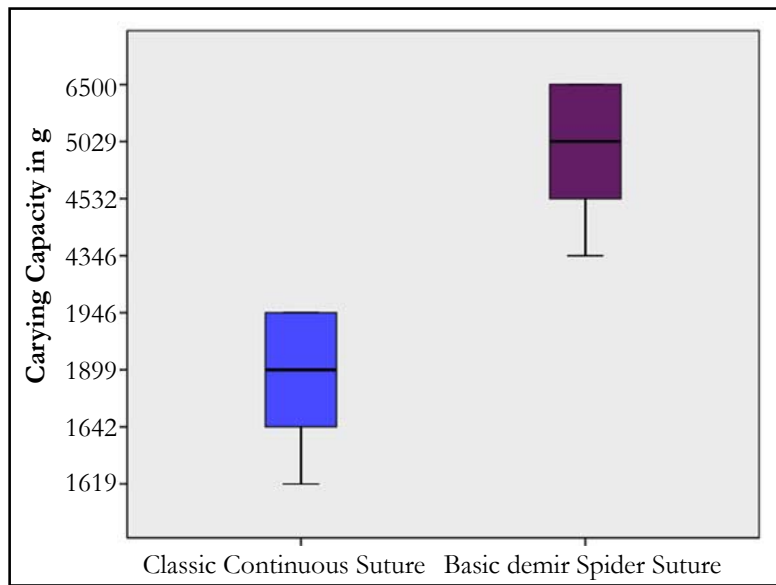

abdomen. The Literature is full of works to find out the best way of closure the fascial wall of the abdomen. Classic known suturing techniques were compared: continuous or interrupted suturing. Materials of the sutures were investigated: absorbable, non-absorbable. But no sweeping progress was observed. From this we should conclude that the material used in these techniques to avoid incisional hernias play no role. Experience from many incisional hernia surgeries shows that the quality of the fascia with the pulling forces acting on it plays an essential role in the development of incisional hernia. The sutures hold, but the fascia does not. The new suturing technique with the inner circular suture absorbs the force acting on the puncture sites of the fascia and distributes it to the entire length of the suture. The circular arrangement of the inner suture allows traction to prevent 
Table 1. Experimental results of carrying capacities of two paper being sutured together in Gram.

\begin{tabular}{|c|c|}
\hline Variable & $\mathrm{N}=5$ \\
\hline Classic Continuous suture & \\
\hline Median in $\mathrm{g}$ & 1777 \\
\hline Range in $\mathrm{g}$ & $1613-1946$ \\
\hline Basic Demir Spider suture & \\
\hline Median in $\mathrm{g}$ & 5102 \\
\hline Range in $\mathrm{g}$ & $4346-6500$ \\
\hline
\end{tabular}

incisional hernia, which is to some extent is independent of fascia quality.

This could be impressively reproduced on a "paper thin fascia" on this experimental setup presented here. The carrying capacity of the sutured paper raised on average from $1777 \mathrm{~g}$ to $5102 \mathrm{~g}$. That meant an increase $287 \%$.

The spider suture could open a door in the surgeon's mind to tailor the abdominal wall at the closure. Depending on the quality of the fascia and the forces acting on it (keyword: body mass index), the surgeon could perform the simple basic spider technique or the extended spider technique, which is shown in Figure 3. Subsequent clinical studies are needed to show the advantages of this novel technique in primary laparotomy closure, incisional hernia repair, umbilical, epigastric hernia repair or in rectus diastasis etc.

\section{Acknowledgements \& Declarations}

Thanks to Mr. Koray Demir, who took the pictures during the experiment.

\section{References}

[1]. Burger JW, Luijendijk RW, Hop WC, Halm JA, Verdaasdonk EG, Jeekel J. Long-term follow-up of a randomized controlled trial of suture versus mesh repair of incisional hernia. Ann Surg. 2004 Oct;240(4):578-83; discussion 583-5. PubMed PMID: 15383785.

[2]. Bloemen A, Van Dooren P, Huizinga BF, Hoofwijk AG. Randomized clinical trial comparing polypropylene or polydioxanone for midline abdominal wall closure. Br J Surg. 2011 May;98(5):633-9. doi: 10.1002/bjs.7398. PubMed PMID: 21254041.

[3]. Schumpelick V, Arlt G, Klinge K. Hernia Surgery-Care of umbilical hernia and incisional hernia. German Physicians' Bulletin-Physician's BulletinIssue A. 1997; 94(51):3471-5.

[4]. Fink C, Baumann P, Wente MN, Knebel P, Bruckner T, Ulrich A, et al. Incisional hernia rate 3 years after midline laparotomy. Br J Surg. 2014 Jan;101(2):51-4. doi: 10.1002/bjs.9364. PubMed PMID: 24281948. 